\title{
On unification, superstrings, and geometry
}

\section{by Yang-Hui $\mathrm{He}^{*}$}

Since the dawn of civilisation, humanity has passionately attempted to understand the natural world - space, time, and all things visible and invisible. This ideal of organizing the myriad of natural phenomena into as few governing laws as possible, this ideal of "unification", seems very much embedded into the subconscious of human aesthetics. Every great culture has a unique approach, with apparent crosscultural similarities. The Chinese archetype is best represented by the concepts of the Five Elements in the "I-Ching" ("Book of Changes", circa 2nd Millennium BC). In "Tao Te Ching" of Lao Tzu ("The way of the Tao", circa C6th BC), the adage "The Tao begets Unity, and Unity begets Duality, and Duality begets Trinity, and Trinity begets all" well summarizes this perspective. The Greek archetype is well exemplified by the "Atomic Theory" of Democritus (circa 460-370 $\mathrm{BC}$ ) or the Five Elements of Aristotle (384-322 BC). Indeed, we could think of these philosophical musings as the beginnings of modern science.

However, our story is on the desires of unification from the point of view of Natural Science. The scientific reductionist approach is what the philosopher Carl Hemple (1907-97) describes as the DeductiveNomological (or D-N) Model. Here, a law, or nomos, forms the basis of a scientific principle, from which explanations of natural phenomena can be deduced. It could be said that this deductive methodology is what we today call Theoretical Physics, and the forefather of this noble subject is indisputably Sir Isaac Newton (1642-1727). The astute reader would question why has it taken 2000 years between the great philosophers and Newton? Indeed, the path of Science is a long one, the conception of a unified un-

*City University, London, United Kingdom; NanKai University, Tianjin, China; and Merton College, University of Oxford, United Kingdom

E-mail: hey@maths.ox.ac.uk derstanding of Nature is a tortuous and perhaps infinite road. In these intervening twenty centuries, humanity has made fantastic progress: The geometry of the West and the algebra of the East have laid the foundations of mathematics; "Science" - as a logical and experimental method emphasizing the observable, the falsifiable and the repeatedly testable - has gradually grown out of "Philosophy" to become the best tool to understand our Natural universe. Yet, we must also acknowledge that it is only after the Renaissance and its brain-child the Early Modern Age (circa C17th-18th), that humanity had developed a drastically new understanding.

One could argue that the crowning achievement of this new age is Newton and his opus magnum "Philosophiae Naturalis Principia Mathematica" (The Mathematical Principles of Natural Philosophy, published 1687). It is well known that in this book, Newton proposed his three Laws of Motion and his universal gravitation. However, what is more important is the philosophical optimism that it had brought to us all: we can start with a handful of equations, utilize the rigours of mathematics, and deduce - whereby explain - countless observations. For example, one can see that Second Law $\vec{F}=m \vec{a}$ combined with universal gravitation $\vec{F}_{G}=\frac{G_{N} m_{1} m_{2}}{|\vec{r}|^{2}} \vec{e}_{r}$ are only two tiny strings of symbols, but thence can be accurately described and predicted the precise motion of an apple falling to the ground or the trajectory of a planet around the sun. The details need not trouble a nonmathematician. By using modern notation of calculus and geometry, a mere page or so of mathematics suffices to deduce, from these two short equations, such data as the elliptical orbits, the speed and period of revolution of the planets etc., data which our ancestors could only ascribe to the gifts of gods. In other words, after Newton, not only can mankind describe and measure Nature, but more importantly, we can 
use the principles of mathematics to deduce the plenitude of natural observations from only a handful of fundamental principles. What is more fitting than this to be called "unification"?!

Indeed, the unifying principle, as clearly stated in the title of Newton's masterpiece, is mathematics. What Newton gave humanity is the legacy of theoretical, or mathematical, physics. It is no wonder that the great poet Alexander Pope (1688-1744) left, as the epitaph on the magnificent monument to Newton in Westminster Abbey, London, the immortal couplet:

Nature and Nature's laws lay hid in night:

God said, "Let Newton be!" and all was light.

\section{Unification}

It has been four centuries since Newton, and theoretical physics has taken her great leaps. Great minds have used the tools of mathematics to reduce and deduce our observable universe ${ }^{1}$ into smaller and smaller sets of principles. Today, theoretical physics can proudly say that we have essentially reduced all of reality to two sets of equations:

1. The Macroscopic: This is the realm of galaxies, stars and heavenly bodies and can be entirely described by the theory of General Relativity of Albert Einstein (1879-1955). Even Newton's universal gravitation is only a special case and approximation. In modern notation, we can write

$$
S[g]=\int d^{4} x \sqrt{-g} R,
$$

which is called the Einstein-Hilbert action.

2. The Microscopic: This is the realm of elementary particles, such as electrons, photons and nucleons, which constitute all matter and can be incorporated into the discipline of Quantum Field Theory. Electromagnetism, the weak and strong nuclear forces are all special cases thereof. In modern notation ${ }^{2}$, we can write

$$
\begin{aligned}
L= & -\frac{1}{4} F_{\mu v} F^{\mu v}+i \bar{\Psi} \not D \Psi+\text { h.c. }+\Psi_{i} Y_{i j} \Psi_{j} \Phi \\
& + \text { h.c. }+\left|D_{\mu} \Phi\right|^{2}-V(\Phi) ;
\end{aligned}
$$

This we call the Standard Model.

From the macroscopic to the microscopic, from galaxies to electrons, all motion, action and change, can in pinciple be deduced from the above two small strings of symbols. That the D-N Model could reach this extent in 400 years is truly astonishing. Of course, now

\footnotetext{
${ }^{1}$ There is still the mysterious "dark matter" whose observation currently eludes us.

${ }^{2}$ As a topical reminder, the symbol $\Phi$ refers to the Higgs particle which has of late caused international sensation from CERN.
}

the details of the mathematical steps far exceed a few pages. Moreover, it is obvious that in practical day to day life, if one wished to compute the motion of a falling apple, there is no need to use quantum theory to track the individual motion of each atom under the laws of gravity. However, the emphasis is on "in principle": We can, given large enough computing power, reduce all that which encompasses us to these two sets of elegant equations.

If "Unity" is our end, then the reader should be disturbed: why two sets of equations instead of one? Is the macroscopic so incompatible with the microscopic?! Indeed, if Newton could unify the treatment of a falling apple with that of revolving planet with such ease, could we not also unify General Relativity with Quantum Field Theory? There are two fundamental reasons for this difficulty:

1. The gravitation of an ordinary object is far less strong than such forces as electromagnetism and nuclear forces. In our universe, only within blackholes or shortly after the moment of creation as the Big Bang can gravity be comparable to the quantum forces;

2. General Relativity has shown us that gravity is, in fact, not even a "force" per se, but is rather the intrinsic curvature of the space and time.

It is ironic that the first universal force which Newton was able to introduce to mankind has become our most baffling one.

The great Einstein of course was fully aware of the importance of the problem. In the middle of the last century, he already stated that were we to unify gravity with quantum theory, then the great "unifying" purpose of physics would be attained. He called this hypothetical theory of unification "the Theory of Everything", or ToE for short. Einstein spent the last decades of his life in vain confronting this formidable challenge. Story has it that even upon his deathbed in Princeton in 1955, Einstein still had his attempts of finding ToE clasped in his hands.

A challenge which defied even Einstein is clearly a difficult one. Though the dark shadow of abandoning our quest for unification haunts us, we should always be mindful of Einstein's own words of wisdom:

The most incomprehensible thing about the universe is that it is comprehensible...

\section{Superstrings}

In the 70 s and 80 s of the last century, a major break-through was led by Michael Green (1946-), John Schwarz (1941-), Edward Witten (1951-) et al. (interestingly, Green and Witten respectively are the successors of Newton and Einstein, quite literally: 
Green is currently the Lucasian Chair in Mathematical Physics at the University of Cambridge and Witten is professor at the Institute of Advanced Study, Princeton and a recipient of the Einstein Prize). They proposed that the reason why gravity cannot be unified with quantum forces is a prejudice which science had held for centuries: that the constituent particles of Nature, such as electrons or photons, are point particles.

The concept of point particles can be traced to at least Newton. In classical Newtonian physics, the fundamental elements are point-like: they have mass but no extent. Even quantum physics, being a theory of waves, is still based on waves induced by point-like particles: for example photons, or light, are points with neither size nor mass, while electrons are points with mass but no size. ${ }^{3}$

This is an audacious hypothesis, no less than when Einstein proposed the space-time continuum or when quantum mechanics proposed wave-particle duality. The advancement of science, especially physics, is always in this spirit: whenever a problem or contradiction arises, the solution rests in valiantly forwarding an earth-shattering new principle which breaks away from previous notions and prejudices. Philosophers call such scientific revolutions paradigm shifts.

If not a point, what then? This is simple, the next object after a point comes a line. Thus, if we studied the motion, the quantum wave, etc., of a line, what shall we obtain? Surprisingly, we obtain the vibration patterns of all particles and all interactions, including gravity! In other words, like all paradigm shifts in the history of science, this revolutionary proposal seemed to have resolved our issue, and we appear to have a unified ToE! We call this line, or string, the superstring, and the theory, superstring theory ${ }^{4}$.

All things in the universe, space and time, thus become different representations, or vibration modes of the superstring. How marvellously beautiful is this paradigm of unification: our world as a harmonious cosmic string symphony.

Now that we have achieved Einstein's dream, what then is there left to accomplish?! Of course, nothing is as simple. Superstring theory has two fundamental problems of her own:

1. How long is the superstring? Because gravity was comparable to quantum forces only in the beginnings of the Big Bang, the size of the superstring is on the scale of that size of the universe a fraction after creation. One can compute that this

\footnotetext{
${ }^{3}$ What we commonly call the "radius" of an electron is only an effective approximation.

${ }^{4}$ The "super" is a double entendre for both the greatness of the theory and a technical concept called supersymmetry on which the theory relies.
}

size - the so-called Planck Length - is approximately $10^{-33}$ meters, which is a smaller than the currently smallest distances mankind can probe - as being investigated by the LHC experiment at CERN - by $10^{10}$ fold (i.e., 10 billion times). Thus, it is unimaginable that we can directly observe the superstring any time soon.

2. Although string theory is incontestably still the best candidate, among a few parallel proposals, for unification, it achieves this marriage between gravity and quantum theory only in the background of 10 dimensions of space-time. ${ }^{5}$ In other words, even though we have theoretically attained a mathematically consistent ToE, she can only be realized in 10 dimensions. However, as we all know, our reality is clearly four dimensional: three of space and one of time.

\section{Geometry}

The first problem is not a serious one. In the history of physics, epoch-making revolutions often had theory preceding experiment. This is a key difference between theoretical physics and other sciences. Experimental science can quantify and generalize from observable data, and extract mathematical principles therefrom. However, theoretical physics does not only conform to this principle, but, perhaps more importantly, she is capable of predicting experimental data based purely on the elegance of mathematics. Neptune must exist because Newtonian physics is beautiful; light must bend on passing by the sun because General Relativity is beautiful; varying magnetic fields must product an electric field because Maxwell's equations are beautiful... Such examples abound.

This guiding principle of beauty almost forms the basis of the correctness in the sense of the D-N Model. Likewise, the word "must" is the key to unification: we are compelled by the simplicity and elegance of the mathematical principle, the principia mathematica. It is appropriate to say that theoretical physics should be a champion knight to the Queen of Mathematics. As far back as the Medieval philosophers, there had been the poignant saying that

"God is the ultimate mathematician".

The importance of mathematics in the Natural world is self-evident. Theoretical physics not only confirms the fact that mathematics is the best language for describing Nature, perhaps one could go as far as saying that Nature herself is only a manifestation, an illustration, of Mathematics.

\footnotetext{
${ }^{5}$ There is theoretical evidence that superstring theory is, in fact, the low energy limit of an 11-dimensional formalism called M-theory.
} 
P. A. M. Dirac (1902-84), after winning the Nobel Prize for predicting the existence of anti-matter based purely on the need for symmetry in his equations, famously said,

"sometimes it is more important to have beauty in one's equations than to have them fit experiment..."

Though this point of view may be a little extreme, we can fully appreciate the historic pretext under which Dirac could be so confident. We need to emphasize that "mathematical elegance" may not be as subjective as one might imagine. A successful theory in theoretical physics must not only be self-consistent, almost as importantly, she often leads to unexpected advances in pure mathematics. As much as Newton's laws of motion developed calculus, or Einstein's gravity, differential geometry, or quantum field theory, algebraic geometry, so too has superstring theory revolutionized many branches of modern mathematics. Even though the theory has yet to be experimentally tested and hence produce Nobel Prizes, related ideas have produced several Fields Medals (the highest honour in mathematics). Witten himself (the first physicist in history to win the Fields Medal) once praised:

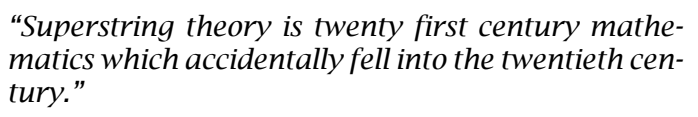

Therefore, for such a consistent theory which can revolutionize mathematics not yet to have experimental evidence is not a fundamental problem. We only need to be patient.

The second problem is an important technical hurdle. But as we shall soon see, this is really a gift in disguise. First, to predict the dimensionality of spacetime is unprecedented. The prediction is a concrete one: our universe is 10 dimensional. There are currently groups at CERN testing this hypothesis. One can only imagine the repercussions if this is verified!

Of course, the world around us is undoubtedly 4 dimensional, where then are the missing $10-4=6$ spatial dimensions?

Given the size of the superstring, this is actually not too difficult to resolve. Suppose an ant is crawling on a thin tree branch, for an observer far away, its world seems to be $1+1=2$ dimensional (it has one dimension of time and one dimension of space along the tree branch). However, for the ant, its world is $2+1$ dimensional (one dimension of time, plus one spatial dimensional along the branch, plus one tiny curled up dimension around the branch). If the ant can crawl into the branch, then there would be one more spatial degree of freedom, giving us the familiar $3+1=4$ dimensions of space-time. In summary, the reason we have not seen 6 missing spatial dimensions is because they are simply too small, as small as the Planck scale which we mentioned earlier, which is far smaller than any machine can directly detect today.

Thus, the universe of the superstring is 4 relatively large dimensions of space-time, and on each point of this familiar set-up, there is a tiny curled up 6-dimensional space. We call this scenario "compactification", it is as if we have made the 6 extra dimensions small and compact. ${ }^{6}$

Of course, not any 6-dimensional shape would do. We must impose strigent physical constraints and different 4-dimensional universes require different 6-dimensional geometries. Conversely, the geometrical properties of 6 dimensions determine the manifestations of the observable 4-dimensional universe. Our unified ToE is thus a geometrical one, thereby pushing the geometrical vision of the Standard Model and quantum field theory - as advocated by S. S. Chern (1911-2004, one of the greatest geometers of the twentieth century), C. N. Yang (1922-, Nobel Laureate in physics), M. Atiyah (1929-, Fields Medalist and former Master of Trinity College, Cambridge) et al. - to new heights.

Years of research in 6-dimensional shapes have shown us that the simplest and perhaps the most elegant possibility is a geometry called Calabi-Yau manifolds, named after E. Calabi (1923-, professor at University of Pennsylvania) and the famous geometer S.-T. Yau (1949-, professor at Harvard University and Fields Medalist). The word "manifold" is a technical one; in mathematics we call a geometrical shape of an arbitrary dimension, not necessarily flat but which can be patched up from flat pieces, a manifold.

What is a Calabi-Yau manifold? Let us start with dimension 2. Two dimensional manifolds are surfaces, such as the surface of a ball. In two dimensions, there is only one (compact and without boundary) Calabi-Yau manifold: it is the surface of a doughnut. Even though the doughnut - with which we are well familiar - has a hole, its surface is a continuous, smooth "manifold" of dimension 2. This surface is called a "torus" in mathematics. If our observable universe were 8 dimensional, then the problem would be quite simple: the 10-dimensional world of superstring can be compactified on the 2 dimensions of the torus and we would get our $10-2=8$ dimensional world, whose physics would be determined by the geometry of the unique torus. However, our universe is 4 dimensional, and we need to seek 6-dimensional Calabi-Yau geometries. As a technical aside on nomenclature, we always refer to a Calabi-Yau manifold by its complex, rather than real, dimension. Thus, just like a complex number has two components, a real and an imaginary, we need

${ }^{6}$ There is an alternative resolution which proposes the extra 6 dimensions to be transverse to a 4-dimensional slice of the total 10 dimensions and the physics is trapped on this slice; this is called the "brane-world". 
to pair up the 6 dimensions, and call it a Calabi-Yau three-manifold, or "Calabi-Yau Threefold".

Hence, the key to our unification scheme is the search for Calabi-Yau threefolds. Indeed, the simplest Calabi-Yau threefold is the 6 real-dimensional doughnut. Unfortunately, there is an abundance in 3 complex dimensions: so far, mathematicians and physicists have identified over a billion different CalalbiYau threefolds. Since each threefold gives a different 4-dimensional universe, which one is correct? That is, which one gives a universe just like our own? Finding the "correct" Calabi-Yau threefold is part of the so-called vacuum degeneracy problem of superstring theory and currently poses as one of the greatest theoretical challenges.

One of the chief directions of my own work is to harness the power of cutting-edge developments in geometry, field theory and large-scale computing to seek, as if trying to find a needle in a haystack, Calabi-Yau threefold structures which give rise to the Standard Model. Intriguingly, collaborative work with B. Ovrut et al. at the University of Pennsylvania and P. Candelas and A. Lukas et al. at the University of Oxford over the last decade has shown that the geometries which give anything akin to our world is extremely rare. ${ }^{7}$ Perhaps this is hinting to us a ge- ometrical selection principle of our universe among the plethora of possibilities.

Furthermore, the Standard Model as we know it has quite a few seemingly God-given constants, such as the mass of the electron, the 3-generation structure of the elementary particles, etc. In superstring theory, these parameters must be representations of a geometrical property. Thus, our goal is not only to find our universe amongst geometrical alternatives, but more importantly, to attempt to isolate the origin of these constants.

The road ahead of us is a long one. Yet thousands of years of philosophy and four centuries of theoretical physics have given us tremendous hope. With great optimism humanity can say that our path is enlightened and the scenery, beautiful.

The great Kepler (1571-1630) once said that " $U b i$ materia, ibi geometria" (where there is matter, there is geometry). That superstring theory should reduce the unified theory of Nature to a problem in the intricacies of modern geometry is precisely in the spirit of this insight. As we stride into the twenty first century, what await us are indubitably deeper physics and more beautiful mathematics.

\footnotetext{
${ }^{7}$ The interested reader is referred to a popular-magazine account in the Jan, 2008 issue of The New Scientist.
} 